\title{
Quantum dynamics in high codimension tilings: from quasiperiodicity to disorder
}

\author{
Julien Vidal, ${ }^{1}$ Nicolas Destainville, ${ }^{2}$ and Rémy Mosseri ${ }^{1}$ \\ ${ }^{1}$ Groupe de Physique des Solides, CNRS UMR 7588, \\ Universités Paris 6 et Paris 7, 2, place Jussieu, 75251 Paris Cedex 05 France \\ ${ }^{2}$ Laboratoire de Physique Théorique, CNRS/Université Paul Sabatier, \\ 118 Route de Narbonne, 31062 Toulouse Cedex 04 France
}

\begin{abstract}
We analyze the spreading of wavepackets in two-dimensional quasiperiodic and random tilings as a function of their codimension, i.e. of their topological complexity. In the quasiperiodic case, we show that the diffusion exponent that characterizes the propagation decreases when the codimension increases and goes to $1 / 2$ in the high codimension limit. By constrast, the exponent for the random tilings is independent of their codimension and also equals $1 / 2$. This shows that, in high codimension, the quasiperiodicity is irrelevant and that the topological disorder leads in every case, to a diffusive regime, at least in the time scale investigated here.
\end{abstract}

PACS numbers: 61.44.Br, 71.23.-k, 71.23.Ft

It is now well established that quasiperiodic order has a strong influence on the quantum dynamics of wavepackets. Indeed, the nature of the eigenstates in quasiperiodic systems, which are neither spatially extended (as in periodic systems) nor localized (as in disordered systems) but critical, is often responsible for a sub-ballistic motion. Although most of the studies about this anomalous diffusion concern one-dimensional systems such as the Fibonacci or the Harper chain, there has also been a great interest for the, more physical, higher-dimensional ones 1, 2, 3, 4, 5]. However, the parameters that determine the characteristics of the long time dynamics, such as the diffusion exponent $\beta$, remains misunderstood.

In this paper, we investigate the quantum dynamics of wavepackets in two-dimensional quasiperiodic tilings built with De Bruijn grid method [6, 7]. This construction allows us to change easily the codimension of the structures that determines the number of different tiles and thus, in a sense, its complexity. The codimension reveals itself as a fundamental quantity for the dynamics since the diffusion exponent decreases when the codimension increases and converges towards $1 / 2$ in the high codimension limit. We also analyze the influence of phason flips that consists in turning the quasiperiodic tiling into a random one without changing the stœchiometry of the tiles (see Fig. (1). We show that contrary to what was claimed in Ref. 1] for the octagonal tiling, the phason disorder do not fasten the spreading but, always slow it down towards a diffusive regime.

The De Bruijn grid algorithm [6, 7] relies on two steps. First, we define $D$ families of $N_{l}$ equally spaced parallel lines, rotated one from the other by an angle $2 \pi / D$. These $D \times N_{l}$ lines form a grid and separate different connected regions of the plane called cells of the decomposition. Second, we dualize this grid by associating a tiling vertex to each cell and connecting any vertices associated to two cells separated by a line.

This algorithm is simpler to implement in two dimensions than the standard Cut-and-Project 8, 9,

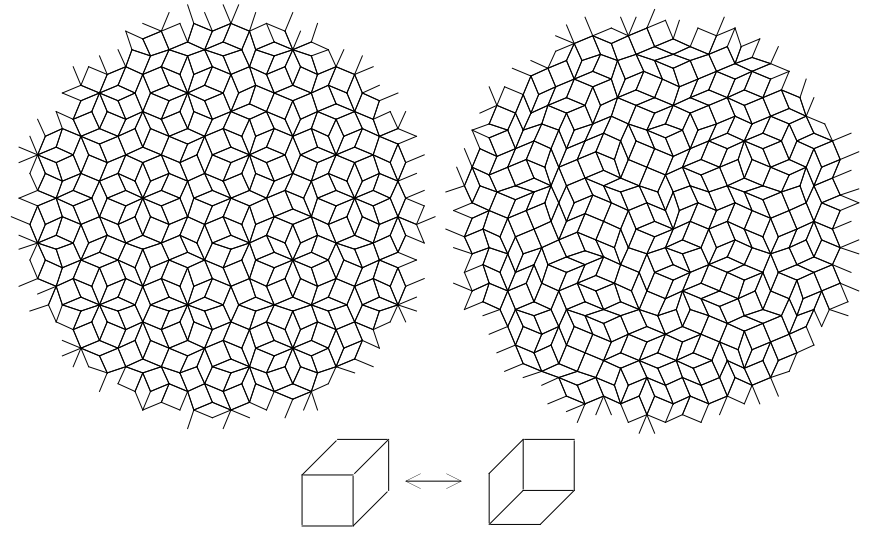

FIG. 1: Left: Quasiperiodic tiling built with the grid method with $D=4$. Right: Random tiling obtained from the quasiperiodic tiling by elementary flips (center). Such transformations conserve the stœchiometry of the tiles but destroy quasiperiodicity and change the coordination number of the sites.

10] method where tilings are generated from highdimensional spaces. In this latter approach, the codimension of the tiling is defined by the difference between the dimension $D$ of the initial lattice in which the points are selected, and the dimension $d$ of the tiling. For twodimensional tilings, the Cut-and-Project method requires the analyzis of a $(D-2)$-dimensional (perpendicular) space whose complexity increases very quickly with $D$ so that, in practice, this method is not tractable beyond $D=5$. By contrast, in the grid method, a tiling of codimension $(D-2)$ is straightforwardly obtained by choosing $D$ families of lines. Note that for $D=2$, the grid method builds the usual square lattice, and, for $D=3$, it builds the well-known (periodic) dice lattice that displays interesting properties when embedded in a magnetic field [11]. Nonperiodic (quasiperiodic) tilings are thus obtained for $D \geq 4$. The number of lines $N_{l}$ determines the size of the 
tiling since the number of sites is $N_{s} \simeq N_{l}^{2} D(D-1) / 2$. However, with this method, different tilings can be obtained depending on the relative position of each line families. Here, we have focussed on a generic family of tilings and checked that our results were weakly sensitive to this dephasing choice. In addition, to avoid spurious effects due to fixed boundary conditions 12] $\left(N_{l}\right.$ is finite), we have isolated a circular central cluster of about $10^{6}$ sites, which is quasiperiodic.

The dynamics is given by a standard tight-binding Hamiltonian:

$$
H=-\sum_{\langle i, j\rangle} t_{i j}|i\rangle\langle j|,
$$

where $|i\rangle$ denotes a localized orbital on site $i$ and where the hopping term $t_{i j}$ equals to 1 if $i$ and $j$ are nearest neighbors and 0 otherwise. Of course, the time evolution of any wavepacket is directly obtained by diagonalizing $H$, but unfortunately, exact diagonalizations are restricted to rather small system size $(\sim 10000$ sites $)$. Since we are interested in the long time behavior of the dynamics that requires large systems, we have used an approximate method, the Second Order Differencing Scheme [13], which consists, for a given initial state $|\psi(0)\rangle$, in writing:

$$
|\psi(t+\Delta t)\rangle=|\psi(t-\Delta t)\rangle-2 i \Delta t H|\psi(t)\rangle .
$$

Practically, a time step $\Delta t=0.05$ is sufficient to get a good accuracy and all our results have been obtained with this value. Note that despite the very low order of this development for the evolution operator $e^{i H t}$, this algorithm is actually an very efficient tool (see for example [14]).

The time evolution of a wavepacket can be characterized by several observables. Here, we focus on the mean square spreading defined by:

$$
\Delta R^{2}(t)=\left\langle\psi(t)\left|\hat{R}^{2}\right| \psi(t)\right\rangle-[\langle\psi(t)|\hat{R}| \psi(t)\rangle]^{2},
$$

where $\hat{R}$ is the position operator, the origin being taken at the center of the cluster. The diffusion exponent $\beta$ is defined by the long time behavior of $\Delta R^{2}(t) \sim t^{2 \beta}$. A priori, nothing prevents from getting a different asymptotic regime but, actually, $\Delta R^{2}$ often seems to be rather well described by a power-law at large time. The case $\beta=1$ obtained for periodic potential defines a ballistic propagation whereas $0<\beta<1$, observed in many quasiperiodic systems, corresponds to a sub-ballistic spreading. Note that the diffusive motion $(\beta=1 / 2)$ is obtained in several situations among which the three-dimensional Anderson model in the metallic part of the spectrum. A crucial issue is to understand the parameters which determine the value of this exponent.

As already noticed in the octagonal tiling 1] for wavepackets initially localized on a single site, $\beta$ strongly depends on this site. In the Labyrinth tiling [3] and in the generalized Rauzy tilings [4 where energy-filtered wavepackets have been studied, $\beta$ has also been shown to be energy-dependent. In the present work, we do not claim to give a precise characterization of the diffusion exponent for each tiling but we wish to investigate the behavior of $\beta$ with respect to the codimension. Therefore, we have chosen to consider random phase states initially spread over a finite portion of the cluster (typically $1 \%$ ). This leads to an exponent which is "averaged" over the total density of states. We have considered several configurations of the phases and several initial radius, and checked that although $\beta$ is different from one state to another, it fluctuates of less than $5 \%$. In addition, since we have finite size systems with open boundary conditions, the propagation must be stopped when the wavepacket reach the boundaries. As a criterion for this maximum time $t_{\text {max. }}$, we have chosen the time for which the presence probability integrated over all the border sites reaches $1 / N_{s}$. Typically, this leads to $t_{\max } \simeq 300$. As usual, we can never ensure that the real asymptotic regime (if it exists !) is reached but this time scale certainly gives some hints concerning the long time behavior.

The mean square spreading $\Delta R^{2}(t)$ is displayed in Fig. 22 for quasiperiodic tilings with $D \in[3,6]$. The dice lattice $D=3$ is given as a reference for a ballistic motion $(\beta=1)$. For low codimension tilings, we observe a superdiffusive regime with an exponent that decreases when the codimension increases.

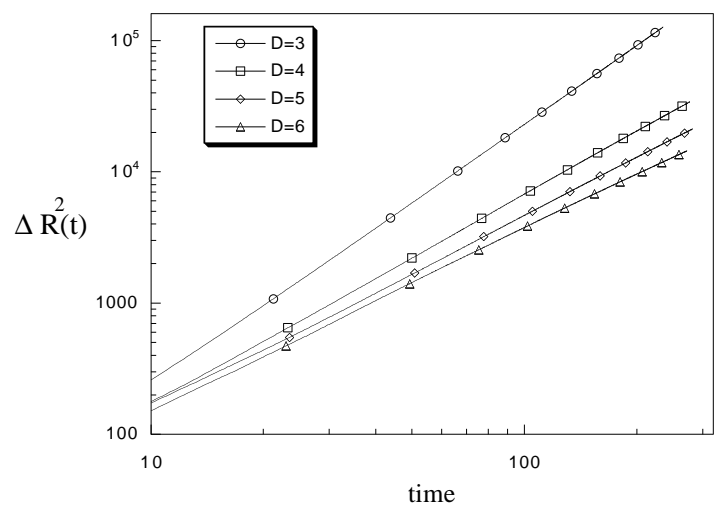

FIG. 2: Log-log plot of $\Delta R^{2}(t)$ for low codimension quasiperiodic tilings.

More precisely, we get $\beta=0.77(1)$ for the octagonal tiling $(D=4), \beta=0.71(1)$ for the Penrose tiling $(D=5)$, and $\beta=0.65$ (1) for the dodecagonal tiling $(D=6)$. Nevertheless, we emphasize that even for these codimensions, the value of the diffusion exponent is not constant over a wide time range and actually slightly decreases when the time increases. This leads us to conclude that either the asymptotic regime is not reached, or the long time behavior is not "strictly" power-law like. 
For higher codimension, the situation is more complicated: no power-law regime can be clearly distinguished since $\beta$ strongly depends on the time range and decreases when the time increases.

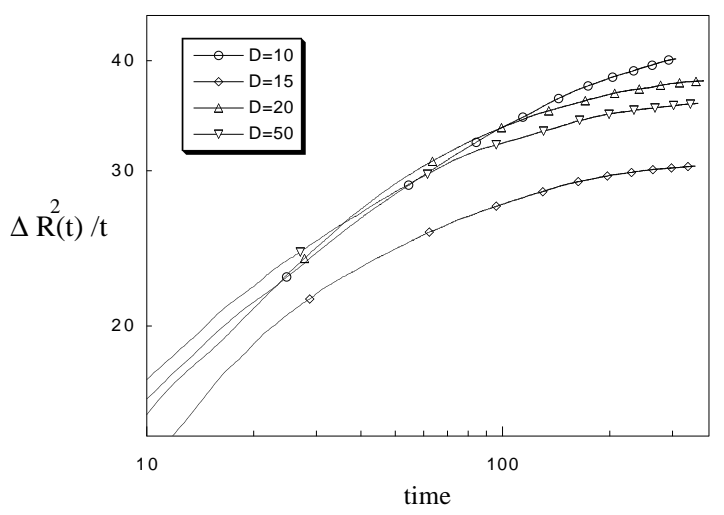

FIG. 3: Log-log plot of $\Delta R^{2}(t) / t$ for high codimension quasiperiodic tilings.

To determine whether it converges towards a asymtotic value (at large time) or not, we have focussed on the behavior of $\Delta R^{2}(t) / t$ which gives us an indication on the super- or sub-diffusive character of the propagation. This is an important issue since in such two-dimensional quasiperiodic systems, no sub-diffusive propagation has ever been reported. As it can be seen in Fig. 3] although no power-law behavior can be extracted in the time range investigated, a strong indication of a diffusive asymptotic regime $\left(\lim _{t \rightarrow \infty} \Delta R^{2}(t) / t=\mathrm{Cte}\right)$ is provided.

These results show that the codimension is an important parameter for the wavepacket propagation. Indeed, noting that for the two-dimensional generalized Rauzy tiling [14] $(D=3)$ one obtains an exponent $\beta=0.95(1)$, it is clear that the higher the codimension, the lower $\beta$. This can be easily understood in terms of the complexity of the structure for the following reasons. An important characteristic of quasiperiodic tilings is the so-called repetitivity property initially studied in two dimensions by Conway. In the class of tilings considered here, it states that for any local environment of linear typical size $L$, a similar environment can be found at a distance $\xi \sim L^{z}$ with $z \geq 1$. In the simplest case $D=4,5$ one has $z=1$. Moreover, when the codimension increases, the number of possible edge orientations at each node is lower or equal to $2 D$ and for a given $L, \xi(L)$ also grows. Consequently, the specific effects of the quasiperiodic order are expected to decrease when the codimension increases. Note that in periodic system, $\xi$ is a constant and in disordered systems, it grows exponentially with $L$.

Nevertheless, these tilings are quasiperiodic and this special order is responsible, at least in low codimension, for a superdiffusive regime $(\beta>1 / 2)$. For higher codimension the quasiperiodicity becomes irrelevant and the exponent $\beta$ seems to converge towards $1 / 2$ which is the value expected for a weakly disordered system in two dimensions (for example a periodic lattice with random impurities or random magnetic fluxes 15], or a disordered quasiperiodic Fibonacci lattice [16]) where such a regime is expected when $\sqrt{\Delta R^{2}}$ is lower than the localization length. Unfortunately, since the asymptotic regime is not reached with the system size studied here, we cannot estimate the codimension dependence of the diffusion constant. However, the results shown in Fig. 3 indicate that this constant does not seem to simply decrease when the codimension increases.

If this analysis is correct, destroying quasiperiodicity in these tilings should also lead to a diffusive regime. To check this assumption and to corroborate our previous statement, we have studied the quantum dynamics in random tilings obtained from the quasiperiodic tilings by making elementary phason flips (about $10^{11}$ ) as shown in Fig. 1for $D=4$. The random flip Markov chain has been studied into detail in the cases $D=3$ and $D=4$ (see [17]), and the number of flips needed to get a random tiling uncorrelated to the initial quasiperiodic tiling is indeed of order $N_{s}^{2}$. Furthermore, this number has been computed numerically in higher codimension tilings and is, in fact, always smaller than $N_{s}^{2} / 2$ [18]. In the present case, this leads to an upper bound of $5.10^{11}$ flips. However, we have checked for $D=4$, that the spreading in tilings with $10^{11}$ and $5.10^{11}$ flips have essentially the same diffusion exponent.

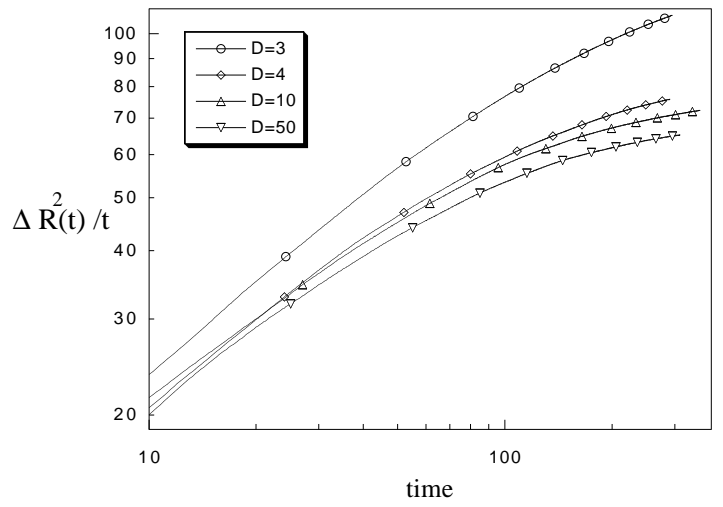

FIG. 4: Log-log plot of $\Delta R^{2}(t) / t$ for random tilings.

As shown in Fig. 4 although no power-law regime can be clearly extracted, the propagation is very similar to those displayed in Fig. 3 for high codimension quasiperiodic tilings. For the octagonal tiling $(D=4)$, this result refutes the conclusion of Passaro et al. claiming that the diffusion exponent increases when a phason disorder is introduced. This contradiction is due to the short time scale investigated in Ref. [1]. Indeed, we also observe that for $t \lesssim 20$, the propagation is faster in the $D=4$ random tiling than in the $D=4$ quasiperiodic 
tiling. However, at larger times, the motion in the random tiling seems to become diffusive whereas, in the octagonal tiling, it remains superdiffusive with $\beta=0.77(1)$ [19]. We have also observed a similar crossover for higher codimension $(D=5,6)$ (see Fig. 5).

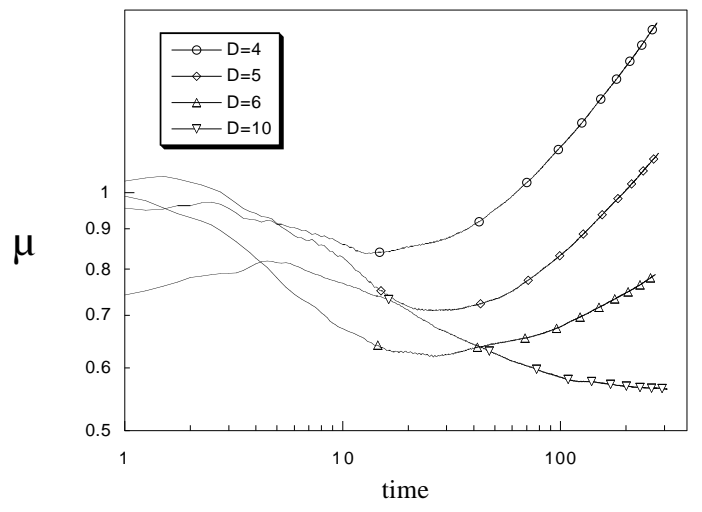

FIG. 5: Log-log plot of $\mu=\Delta R_{\text {quasi. }}^{2} / \Delta R_{\text {random }}^{2}$ as a function of time.

A possible explanation of these two regimes is that, at short times, the local quasiperiodic order is likely responsible for coherent quantum interferences (absent in a random tiling) that slow down the dynamics. In this regime, the topological disorder thus favors, in a sense, the propagation. At large times, the influence of the quasiperiodicity remains the same but a weak localization process becomes dominant for the random tiling and leads to a diffusive regime. Of course, for larger codimension, the situation is different since the asymptotic regime is likely the same both in the quasiperiodic and in the random case. Thus, one expects $\mu=\Delta R_{\text {quasi. }}^{2} / \Delta R_{\text {random }}^{2}$ to become constant at large time and it is actually the case (see Fig. 5 for $D=10$ ).

This study raise interesting questions concerning the nature of the spectrum in quasiperiodic and random tilings. A simple way to characterize the spectral measure of a state $|\psi\rangle$ is to compute the averaged autocorrelation function

$$
C(t)=\frac{1}{t} \int_{0}^{t} d t^{\prime} P\left(t^{\prime}\right) \sim t^{-\alpha},
$$

where $P(t)=|\langle\psi(0) \mid \psi(t)\rangle|^{2}$. If $\alpha=1$, the spectral measure is absolutely continuous, if $\alpha=0$, it is pure point, and if $0<\alpha<1$ it is singular continuous. As previously, for a random phase state, $\alpha$ gives an average information over the total density of states. Unfortunately, in the tilings studied here, there exist strictly localized states known as confined states due to specific local environ- ment (see Ref. 20, 21] for the case $D=5$ ). These states eventually leads to $\alpha=0$ provided the initial random phase state has a nonzero overlap with them. The analyzis of the spectral measure with respect to the codimension thus requires to get rid of these confined states. Further investigations are needed to clarify this point which is certainly of great interest in the understanding of the electronic properties of quasiperiodic systems.

We would like to thank M. Baake and M. Duneau for fruitful remarks about the repetitivity property of quasiperiodic tilings.

[1] B. Passaro, C. Sire, and V. G. Benza, Phys. Rev. B 46, 13751 (1992).

[2] J. X. Zhong and R. Mosseri, J. Phys. C 7, 8383 (1995).

[3] H. Q. Yuan, U. Grimm, P. Repetowicz, and M. Schreiber, Phys. Rev. B 62, 15569 (2000).

[4] F. Triozon, J. Vidal, R. Mosseri, and D. Mayou, Phys. Rev. B 65, 220202 (2002).

[5] J. Vidal and R. Mosseri, Proceedings of ICQ7, edited by F. Gähler, P. Kramer, H. R. Trebin, and K. Urban (Elsevier, Switzerland, 2000), Vol. A294-A296, p. 572.

[6] N. G. de Bruijn, Proc. Konink. Ned. Akad. Wetensch. A 43, 84 (1981).

[7] N. G. de Bruijn, J. Phys. France 47, C3 (1986).

[8] M. Duneau and A. Katz, Phys. Rev. Lett. 54, 2688 (1985).

[9] P. A. Kalugin, A. Y. Kitaev, and L. S. Levitov, J. de Phys. (Paris) Lett. 46, L601 (1985).

[10] V. Elser, Phys. Rev. Lett. 54, 1730 (1985).

[11] J. Vidal, P. Butaud, B. Douçot, and R. Mosseri, Phys. Rev. B 64, 155306 (2001).

[12] N. Destainville, J. Phys. A 31, 6123 (1998).

[13] C. Leforestier et al., J. Comp. Phys. 94, 59 (1991).

[14] J. Vidal and R. Mosseri, Proceedings of ICQ8, cond-mat/0209037 unpublished.

[15] T. Kawarabayashi and T. Ohtsuki, Phys. Rev. B 51, 10897 (1995).

[16] S. Roche and D. Mayou, Phys. Rev. Lett. 79, 2518 (1997).

[17] N. Destainville, Phys. Rev. Lett. 88, 030601 (2002), and references therein.

[18] M. Widom, N. Destainville, R. Mosseri, and F. Bailly, unpublished.

[19] For $D=3,4$, it is possible to have an explicit form for the sites coordinates thanks to a special numbering. Thus, for the Rauzy tiling and the octagonal tiling, we have checked that $\beta$ remains the same up to $1 \%$ for tilings with about $8.10^{6}$ sites!

[20] M. Kohmoto and B. Sutherland, Phys. Rev. B 34, 5043 (1986).

[21] M. Arai, T. Tokihiro, T. Fujiwara, and M. Kohmoto, Phys. Rev. B 38, 1621 (1987). 